\title{
Prevalence and clinical presentation of HIV positive female psychiatric inpatients
}

\author{
H Uys \\ East London Mental Health Unit, Cecilia Makiwane Hospital, East London Hospital Complex, East London, South Africa
}

\begin{abstract}
Objective: Literature on the prevalence and clinical presentation of South African psychiatric HIV positive patients as well as their management is lacking. This study was performed to investigate these parameters. Method: A prospective study to investigate the prevalence and clinical presentation of HIV positive psychiatric female patients admitted to an acute ward (female psychiatric ward Cecilia Makiwane Hospital, East London Hospital Complex) was undertaken for a one year period from June 2009 to May 2010. Additional factors such as demographic profile, associated substance abuse, pre-existing psychiatric disorders and management were also studied. Results: During this one year period 158 female patients were admitted to the acute female psychiatric ward for assessment and treatment. Of these 158 admissions 21 (13\%) were HIV positive, but only 19 (12\%) consented to the study. Ten of the 19 HIV positive patients (53\%) had an Axis I diagnosis of a psychiatric disorder secondary to HIV, most commonly mood disorder (mania) with psychotic symptoms. Nine of the 19 HIV positive patients (47\%) had a pre-existing primary psychiatric diagnosis, most commonly Bipolar Disorder, recent episode mania with psychotic symptoms. The most common psychotic symptoms were grandiose delusions followed by auditory hallucinations, paranoid delusions and visual hallucinations. The most common mood symptom was euphoria. Substance abuse was uncommon amongst the patients in the study group. Sixteen of the 19 patients (84\%) were on antipsychotics, 14 of these 16 patients were on haloperidol. The most commonly used mood stabilizer was sodium valproate. Conclusion: In this study mania with psychotic symptoms was the most common presentation in both the group with a pre-existing primary psychiatric disorder and in the group without a primary psychiatric disorder. The prevalence of HIV positive admitted psychiatric females in this study was $12 \%$. Co-morbid substance abuse was uncommon. Most patients tolerated low doses of typical antipsychotics in combination with a mood stabilizer (valproate being the most common).
\end{abstract}

Keywords: HIV; Female; Psychiatric diagnosis; Hospitalization; South Africa

Received: 14-02-2011

Accepted: 04-1 1-2011

doi: http://dx.doi.org/10.4314/ajpsy.v16il.4

\section{Introduction}

Sub-Saharan Africa remains the region most severely affected by Human Immunodeficiency Virus (HIV). In 2008, infections within sub-Saharan Africa accounted for $67 \%$ of HIV infections worldwide. Women and young girls continue to be affected disproportionately by HIV in sub-Saharan Africa. Women's vulnerability to HIV seems to stem not only from their greater physiological susceptibility to heterosexual viral transmission, but also from factors such as social, legal and economic disadvantages. ${ }^{1}$ The national HIV and syphilis prevalence

\section{Correspondence}

Dr H Uys

East London Mental Health Unit, Cecilia Makiwane Hospital

Private Bag 9047, East London, 5202, South Africa

email: psyc@sainet.co.za survey of South Africa (2007) reports that the national HIV prevalence rate has been stable for several years and evidence now suggests that the epidemic is on the decline. The 2007 national HIV prevalence rate is $28 \%$. It further reports that the Eastern Cape is one of the provinces in which a reduction was seen. ${ }^{2}$

Psychiatrists are regularly confronted by HIV positive patients who may present with new onset psychiatric disorders or an exacerbation of their underlying psychiatric illnesses. Estimates of the lifetime and past prevalence rates of psychiatric disorders in persons living with HIV/Acquired Immunodeficiency Syndrome (AIDS) may differ depending on the sample and comparison groups. Data on HIV- infected women at the South Texas AIDS Center for Children and Their Families showed that over $60 \%$ met the criteria for at least one 
Axis I Disorder with major depression and substance abuse being most common. A wide range of psychiatric disorders can be directly or indirectly associated with HIV infection. These disorders include delirium, HIV associated dementia (HAD), HIV associated mania, minor cognitive motor disorders, adjustment disorders, mood disorder, psychotic disorder, and substance abuse. ${ }^{3}$

Literature on the psychiatric presentation of South African HIV positive patients and their management is lacking. ${ }^{4}$ Given the observation by the author that a significant proportion of females admitted to the female psychiatric ward in the East London Mental Health Unit were HIV positive and that most of these patients presented with mood (manic) and psychotic symptoms - this study was therefore conducted to investigate the prevalence, clinical presentation, and treatment of HIV positive patients in an acute psychiatric ward

\section{Method}

\section{Site/sample}

A prospective study of HIV positive psychiatric female patients admitted to an acute ward (female psychiatric ward Cecilia Makiwane Hospital, East London Hospital Complex) was undertaken for a one year period from June 2009 to May 2010. These patients were known HIV positive patients or were tested during their admission.

\section{Procedure}

The patients were interviewed by the investigator (psychiatrist with 16 years experience), using a standard interview protocol used in the Cecilia Makiwane Psychiatric wards. The following information was elicited during the interview. 1) Demographics, 2) Reason for admission, 3) Clinical presentation, 4) Substance abuse, 5) Past psychiatric history, 6) Family psychiatric and medical history. A Mental status review, including Folstein's Mini Mental Status Examination, was conducted in every patient. A diagnosis was made using DSM IV-TR criteria, and appropriate treatment was subsequently started. In addition, investigations, including CD 4 count, lumbar puncture, and brain scan were performed.

\section{Ethics}

Informed consent for the study was obtained from the participants or a family member and ethics approval was obtained from the East London Hospital Complex's (ELHC) ethics committee (consent forms were available in English and Xhosa). The current policy for HIV testing in the ELHC was followed (that of written informed consent with pre- and post- test counseling). Written consent for HIV testing and study participation was obtained by the investigator once the patients were mentally stable.

\section{Results}

During this one year period, 158 female patients were admitted to the acute female psychiatric ward for assessment and treatment. Of these 158 admissions 21 (13\%) were HIV positive, but only 19 (12\%) consented to the study .The demographics of the HIV positive patients are displayed in Table I. All of the patients were Xhosa-speaking and most were single, unemployed, and with a secondary school education.

\begin{tabular}{|c|c|c|}
\hline \multicolumn{3}{|l|}{ Table I: Demographic data } \\
\hline & N & $\%$ \\
\hline \multicolumn{3}{|l|}{$\begin{array}{l}\text { AGE } \\
\text { Average age: } 35(26-51)\end{array}$} \\
\hline \multirow{4}{*}{$\begin{array}{l}\text { LANGUAGE } \\
\text { Xhosa } \\
\text { English } \\
\text { Other languages }\end{array}$} & & \\
\hline & 19 & $100 \%$ \\
\hline & 0 & ०\% \\
\hline & 0 & $0 \%$ \\
\hline \multirow{3}{*}{$\begin{array}{l}\text { EMPLOYMENT } \\
\text { Employed } \\
\text { Unemployed }\end{array}$} & & \\
\hline & 5 & $26 \%$ \\
\hline & 14 & $74 \%$ \\
\hline \multirow{4}{*}{$\begin{array}{l}\text { EDUCATION } \\
\text { Grade } 12 \text { education (matric) } \\
\text { Secondary school education (but below grade 12) } \\
\text { Primary school education }\end{array}$} & & \\
\hline & 6 & $31,5 \%$ \\
\hline & 10 & $53 \%$ \\
\hline & 3 & $15,5 \%$ \\
\hline \multirow{4}{*}{$\begin{array}{l}\text { MARITAL STATUS } \\
\text { Married } \\
\text { Single } \\
\text { Widowed }\end{array}$} & & \\
\hline & 5 & $26 \%$ \\
\hline & 12 & $63 \%$ \\
\hline & 2 & $11 \%$ \\
\hline
\end{tabular}

Ten of the 19 HIV positive patients (53\%) had an Axis I diagnosis of a psychiatric disorder secondary to HIV. Three of these ten patients (30\%) had a diagnosis of psychotic disorder secondary to HIV, six (60\%) had a diagnosis of mood disorder (mania) with psychotic features secondary to HIV and one (10\%) had a diagnosis of HIV associated dementia (Table II). Five of these ten patients (50\%) presented with their first onset of psychosis at the time of the study period. Four of these patients (40\%) had one previous psychiatric episode. In three of these patients the previous diagnosis correlated with the current diagnosis. In the patient with dementia this was not the case. One of these patients (10\%) had two previous episodes with a similar diagnosis.

Nine of the 19 HIV positive patients (47\%) had a preexisting primary psychiatric diagnosis. The pre-existing diagnosis in all nine patients (100\%) was Bipolar Disorder Type I, and all presented with mood (manic) and psychotic symptoms during their current admission (Table II). Hence it was uncertain whether their current symptoms represented a new disorder secondary to HIV, or whether the symptoms were an exacerbation of their previously diagnosed psychiatric disorder.

\begin{tabular}{|l|l|l|}
\hline \multicolumn{2}{|l|}{ Table II Axis I Disorder } \\
\hline Psychiatric Disorder secondary to HIV & $N(10)$ & $53 \%$ \\
Psychotic Disorder & 3 & $30 \%$ \\
Mood Disorder (mania with psychotic symptoms) & 6 & $60 \%$ \\
Mood Disorder (depression) & 0 & $0 \%$ \\
Dementia & 1 & $10 \%$ \\
\hline Pre- existing primary psychiatric disorder & $N(9)$ & $47 \%$ \\
Schizophrenia & 0 & $0 \%$ \\
Bipolar Disorder Type 1 & 9 & $100 \%$ \\
Unipolar depression & 0 & $0 \%$ \\
Dementia & 0 & $0 \%$ \\
\hline
\end{tabular}


Overall 15 of the 19 (79\%) patients presented with mood (manic) and psychotic symptoms. The most common mood symptom was euphoric mood and the most common psychotic symptoms were grandiose delusions, followed by auditory hallucinations, paranoid delusions and visual hallucinations. The patient with dementia presented with auditory hallucinations without delusions. Only one of the 19 patients had a history of substance abuse (benzodiazepines and analgesics). None of the patients had a history of any illicit substance abuse.

Two of the patients had a family history of substance abuse but no family history of mental illness was elicited. Fourteen patients had a prior psychiatric episode. In terms of medical co-morbidity, three patients had been previously treated for tuberculosis (TB) and one was treated for TB during the study period. One patient had a prior history of Steven Johnson's syndrome and one had a history of Efavirenz-induced hepatitis (Table III).

\begin{tabular}{|l|l|l|}
\hline \multicolumn{3}{|l|}{ Table III: Clinical presentation, co-morbidities and previous } \\
psychiatric history & $N$ & $\%$ \\
\hline & & \\
\hline CLINCIAL PRESENTATION & 9 & $47 \%$ \\
Auditory hallucinations & 3 & $16 \%$ \\
Visual hallucinations & 12 & $63 \%$ \\
Grandiose delusions & 7 & $37 \%$ \\
Paranoid delusions & 10 & $53 \%$ \\
Euphoric mood & 7 & $37 \%$ \\
Irritable mood & & \\
\hline CO-MORBID MEDICAL DISORDERS & 3 & $16 \%$ \\
TB (previously) & 1 & $5 \%$ \\
TB (currently) & 1 & $5 \%$ \\
Drug induced hepatitis & 1 & $5 \%$ \\
Steven Johnson's syndrome & & \\
\hline SUBSTANCE ABUSE & 0 & $0 \%$ \\
IIIicit & 1 & $5 \%$ \\
\hline Over the counter medications and prescription medication & 14 & $74 \%$ \\
\hline PREVIOUS PSYCHIATRIC HISTORY & 0 & $0 \%$ \\
\hline FAMILY PSYCHIATRIC HISTORY & 2 & $11 \%$ \\
\hline FAMILY HISTORY OF SUBSTANCE ABUSE & & \\
\hline
\end{tabular}

All nineteen patients had the following blood tests done: full blood count (FBC), thyroid functions (TFT), liver functions (LFT), urea and electrolytes (U\&E) and syphilis screen (RPR). Additional tests performed were serum vitamin B 12 levels, chest x-ray, brain scan, lumbar puncture (LP), pregnancy test and hepatitis screen. All 19 patients had their CD 4 count checked during their study period. The average CD 4 count of the patients diagnosed with a psychiatric disorder secondary to the HIV was 186 (lowest was 29) and the average CD 4 count of the patients with the pre-existing primary psychiatric disorder was 366 (lowest 57). Nine of the 19 patients (47\%) had a CD 4 count less than 200. The average score of the Folstein's Mini-Mental Status Examination (MMSE) was 26 out of 30, with a lowest score of
11. Four patients scored 30. The group with the pre-existing primary psychiatric diagnosis had a mean MMSE score of 25,6 and the group with the diagnosis of a psychiatric diagnosis secondary to the HIV scored a mean of 26,3. The patient with the lowest MMSE score (1 l) had a diagnosis of HIV associated dementia (see table IV).

\begin{tabular}{|l|l|l|}
\hline \multicolumn{2}{|l}{ Table IV: Abnormal investigations, CD 4 counts and MMSE } \\
scores & N & COMMENT \\
\hline TEST: & 5 & Mildly decreased \\
\hline White cell count & 3 & Mildly decreased \\
\hline Hemoglobin & 2 & $\begin{array}{l}\text { One mildly elevated and } \\
\text { one mildly decreased }\end{array}$ \\
\hline MCV & 2 & Mildly elevated \\
\hline GGT & 4 & Mildly elevated \\
\hline GGT and transaminases & 4 & $\begin{array}{l}\text { Significant generalized } \\
\text { brain atrophy }\end{array}$ \\
\hline Brain Scan & 1 & Average MMSE score \\
\hline DISORDER & Average CD 4 count & 26,3 \\
\hline $\begin{array}{l}\text { Psychiatric disorder } \\
\text { secondary to HIV }\end{array}$ & 186 (lowest 29) \\
\hline $\begin{array}{l}\text { Pre-existing primary } \\
\text { psychiatric disorder }\end{array}$ & 366 (lowest 57) & 25,6 \\
\hline
\end{tabular}

Sixteen of the 19 patients (84\%) were on antipsychotics, 14 of these 16 patients were on haloperidol. The other two patients were on quetiapine and zuclopenthixol depot respectively. The average dose of haloperidol was 2, $9 \mathrm{mg}$. Fifteen patients were on mood stabilizers. The most commonly used mood stabilizer was sodium valproate and the most often used combination was haloperidol and sodium valproate. Two patients were on two mood stabilizers (valproate and lithium). Only one patient's haloperidol was reduced from $5 \mathrm{mg}$ to 2.5 mg as a result of extra-pyramidal side effects. None of the patients were on antidepressants. Seventeen of the 19 patients (89\%) were on anti- retroviral treatment (ART) (Table V). Twelve of these were started on regime 1B (which does not include efavirenz and comprises - nevirapine, stavudine and

\begin{tabular}{|l|l|l|}
\hline \multicolumn{2}{|l|}{ Table V: Psychotropic medications and ART } \\
\hline Psychotropics & $\begin{array}{l}\text { Number of patients on } \\
\text { psychotropic agent }\end{array}$ & $\%$ \\
\hline Antipsychotics, typical (oral) & 14 (Haloperidol) & $74 \%$ \\
Antipsychotics, typical (depot) & 1 (Zuclopenthixol depot) & $5 \%$ \\
Antipsychotics, atypical (oral) & 1 (Quetiapine) & $5 \%$ \\
Antipsychotics, atypical (depot) & 0 & $0 \%$ \\
Antipsychotics ( total) & 16 & $84 \%$ \\
Mood stabilizers (total) & (Lithium and sodium Valproate) & $79 \%$ \\
Antidepressants (total) & 0 & $0 \%$ \\
ART (total) & 17 & $89 \%$ \\
\hline
\end{tabular}


lamivudine) during the study admission whilst the other five were already on ART for periods of longer than 3 months prior to the admission. The work up for ART was done in the psychiatric ward in consultation with the ARV (anti-retroviral) unit situated in the Hospital Complex.

\section{Discussion}

The low figure of HIV positive patients presenting to the acute female psychiatric ward was an unexpected finding. This could be explained by the stringent ethical principles that are applied when testing mentally ill patients. This study also did not investigate how many of the 158 admissions in the study period were actually tested for HIV as at the time of the study there was no accurate record keeping of which patients were tested for HIV or not. The true prevalence of HIV positive admitted patients could therefore be much higher. The reported HIV seroprevalence in psychiatric inpatients range from 0 $59 \%$ with a mean of $10 \%$. There is a clear trend in the increase in the prevalence - before 1996 the seroprevalence was 7, 4\%, after 1996 the mean was $15 \%$. As the prevalence of HIV in young adults in South Africa is close to $20 \%$, with a suspected higher prevalence in mentally ill (most of whom are unable to give informed consent), and with treatment readily available, a new approach to HIV testing should be investigated. ${ }^{5}$ The need to integrate mental health and HIV care is increasing and the role of mental health in ARV programmes is central. ${ }^{6}$

The male to female heterosexual transmission rate has been estimated to be 20 times higher than the female to male transmission rate. HIV prevalence generally peaks at a younger age for women than for men, with peak ages for women between 30 and 34 and between late thirties and early forties for men. Women's vulnerability could stem from both biological and social risk factors. Biological factors implicated are factors which are present and provide direct viral access to the bloodstream, or that cause inflammation which result in greater numbers of target cells, or that facilitate the survival of HIV in mucosa. In addition other risk factors include receptive anal intercourse, genital ulcers, cervical ectopy, other sexually transmitted diseases, hormonal contraceptives, and pregnancy. Numerous social factors can place women at risk. Cultural norms and attitudes may discourage sex education and use of safe-sex method. Poverty could increase the risk through several mechanisms. ${ }^{3}$ Sexual and physical violence also increase the risk. The prevalence of HIV is significantly higher amongst divorced, separated and widowed women as opposed to single or married women. ${ }^{1}$

The average age of the study group was 35 years, which fits in with the peak HIV prevalence rate in women. Fourteen women were unemployed, which further supports the vulnerability theory. All the study participants were black and Xhosa- speaking and therefore from the same cultural background. Attitudes towards sex education, using safe sex, and physical abuse were not specifically documented in the study group. However, contraception was documented and eleven patients used no contraception, five used depot contraceptives and three reported using condoms as a contraceptive measure. All participants were heterosexual and none of these patients were pregnant.

The most common psychiatric and psychological problems encountered in HIV positive women are depression, anxiety, suicide and several psychosocial problems such as stigma, lack of support and coping with several losses. ${ }^{3}$ The prevalence of depression in HIV positive patients ranges from 20 to $37 \%$, which is higher than the general population. ${ }^{7}$ None of our patients were diagnosed with depression and only one patient had a history of substance abuse. The low depression rates in this study could be explained by investigating an inpatient population rather than an outpatient population, as well as the small sample size.

Psychosis is a recognized but, relative to the mood disorders, uncommon psychiatric manifestation of AIDS. HIV- infected psychotic patients exhibit more cognitive deficits than controls without psychotic symptoms. ${ }^{8}$ The following could explain the underlying mechanism for psychotic symptoms in HIV positive patients:

- direct neuropathic effect of the virus on the brain

- secondary to the effect of medication or drug abuse

- secondary to opportunistic infections

- independent psychiatric disorder may emerge coincidentally HIV may precipitate psychosis in a predisposed individual

- The psychological impact of the knowledge of being infected may trigger a psychotic episode

Higher rates of mania have been reported with progression of HIV infection. In early HIV infection 1-2\% of patients experience manic episodes (rate is only slightly higher than that of the general population). This increases to $4-8 \%$ after the onset of AIDS. This has been closely associated with cognitive changes or dementia. The MMSE mean score of patients in this study was 26 (which is regarded as normal) and does not support the above notion that both manic and psychotic episodes in HIV positive patients are associated with cognitive changes. Memory impairment and psychomotor slowing are some of the primary indicators of cognitive problems in HIV associated dementia (HAD). However, the MMSE was designed to screen for Alzheimer's dementia rather than the subcortical dementias associated with HIV. The MMSE does not include timed tasks which assess psychomotor slowing and is therefore not sensitive enough to measure $\mathrm{HAD}$. The following dementia scales are more sensitive to identify cognitive problems in HIV positive persons: The HIV Dementia Scale (HDS), the modified HDS Scale (M $\mathrm{HDS}){ }^{9}$

The International HIV Dementia Scale from Sacktor 2003 (IHDS) is another scale, which can be used to detect cognitive problems and dementia in HIV positive patients, and could even be applied at the primary health care level. ${ }^{10}$

Psychosis occurring in HIV positive patients could be new onset psychosis or an exacerbation of a previous psychiatric illness. The seroprevalence studies of patients with psychosis have found 5\% - 20\% to be HIV positive, 
which suggest that psychosis can increase the risk of HIV. ${ }^{11}$ The diagnosis is more likely to be a primary diagnosis (i.e. not due to the medication for HIV, medical illness or HIV itself) if:

- The immune system is relatively intact

- A family history is present

- A psychosocial stressor is present

- Cognitive impairment is absent

Several studies have reported on the clinical presentation of new onset psychosis in HIV positive patients: Burich et al reported in 1988 on their three patients who presented with symptoms indistinguishable from functional psychosis. These patients presented with affective psychosis and schizophreniform psychosis without any cognitive impairment. Susser et al reported on 12 examples of new onset psychosis in patients between $20-39$ years. The HIV infection was antecedent to the onset of psychosis and it was postulated to be the cause of the psychosis. These patients presented with a variety of psychotic symptoms, high rate of poly substance abuse, various stages of HIV infection and often no encephalopathy. ${ }^{11}$ Sewell et al reported in 1994 on new onset psychosis in 20 patients. Mean age in this group was 35 years, 100\% had delusions, 90\% had hallucinations and $81 \%$ had prominent mood symptoms. Their psychotic patients differed from non psychotic HIV matched controls in having higher rates of substance abuse and a higher mortality rate on follow up. They also had more neuropsychological impairment. ${ }^{12}$ Halstead reported on five HIV positive patients presenting with new onset psychosis. These patients had prominent mood symptoms, delusions, hallucinations and thought disorder. Three of these patients demonstrated no organic features, but three out of the five cases had a history of substance abuse. ${ }^{13}$ Harris et al reported in 1991 that common clinical features noted in HIV positive patients with new onset psychosis included acute or subacute onset, delusions, hallucinations, affective symptoms, bizarre behavior and mild cognitive impairment. ${ }^{14}$

South African studies have reported on first onset psychosis in HIV positive patients. Mashaphu and Mkize reported on a high seroprevalence of $23,8 \%$ among patients presenting with a first onset psychosis. This was an increase from $2 \%$ in the same institution since 1992 (unpublished data S D Chetty 1992). These patients presented mainly with schizophreniform psychosis. There was no conclusive evidence that the HIV itself caused the psychosis, but this aetiology cannot be ruled out. None of these patients had a known co-morbid illness and therefore it supports the theory that new onset psychosis could be part of HIV associated encephalopathy. ${ }^{15}$ Sall et al reported on psychiatric symptoms and disorders in HIV infected (untreated) South African mine workers. Their study group comprised of 38 patients. One half of their patients presented with some form of cognitive symptoms. A substantial number of these patients also presented with psychotic symptoms - delusions were the most common. Affective symptoms were also common in this study group (mania more prevalent than depression). Overall dementia was the most common diagnosis in this group, occurring in almost one third of patients, most presenting with psychotic symptoms. Almost one third of patients presented with psychotic symptoms without cognitive symptoms Substance abuse was common in this study group. The authors argued that in any cases of mania, psychosis or cognitive deficits in a previous healthy individual HIV should be considered. ${ }^{16}$

Janse van Rensburg and Bracken reported on the clinical presentation of HIV positive admitted patients. Their sample consisted of 34 patients (22 females and 12 males). The most common diagnosis was that of psychosis secondary to a general medical condition (not specified whether these were episodes of first onset psychosis or not). Their overall finding was a predominance of psychotic and manic symptoms with a significant amount of substance abuse. ${ }^{4}$ In our study group five out of the 19 patients presented with a first onset psychosis (26\%). All five presented with psychotic symptoms and three with mood (manic) symptoms. None of these patients abused any substances. All of these findings are in keeping with the literature except the low prevalence of substance abuse. The low prevalence of substance abuse could be explained by the small study population, as well as substance abuse being less common amongst females (no males in study population). The history of substance abuse was obtained from the patients as well as from the collateral history and previous notes (if previously admitted).

Overall, 15 of the 19 patients in the current study presented with mood (manic) and psychotic symptoms. The most common mood symptom was euphoria, whereas the most common psychotic symptoms were grandiose delusions, followed by auditory hallucinations, paranoid delusions, and visual hallucinations.

Persons with severe mental illness are at risk to develop HIV infection. The seropositivity in this group is estimated to be between 3\% -23\%. The most common Axis I diagnoses in this group are schizophrenia, schizoaffective disorder and bipolar disorder. ${ }^{3}$ These patients are often involved in increased risk taking behavior which includes inappropriate sexual behavior and substance abuse. Our study group reported that the most common pre-existing diagnosis was that of bipolar disorder. Our study did not confirm the co- morbid substance abuse disorder as reported by the literature.

Sixteen of our 19 patients were on antipsychotics (haloperidol being the most commonly-used agent) and 15 patients were on a mood stabilizer (79\%). Valproate was the most commonly used mood stabilizer. Two patients were on two mood stabilizers (lithium and valproate). The average dose of haloperidol was $2.9 \mathrm{mg}$, which is lower than in the study by Janse van Rensburg and Bracken where the average dose was 7, $5 \mathrm{mg}$. In their study $50 \%$ of their patients were on valproate. ${ }^{4}$ Vitiello et al reported on the use of psychotropics in HIV positive patients in the United States. Antidepressants were the most commonly prescribed psychotropic followed by anxiolytics. ${ }^{17}$ None of our patients were on antidepressants. This could be explained by our patients presenting mainly with manic, psychotic and behavioral symptoms which necessitated the use of antipsychotics and moodstabilizers, and hospitalization.

Our study population reflects that all patients (except two) were on ART, and twelve patients were started on ART during their psychiatric admission. 


\section{Conclusion}

At least 19 of 153 (12\%) female patients admitted over a period of twelve months were HIV positive. Although this does not reflect the true prevalence of HIV positive patients admitted to this unit (due to the fact that the actual number of patients tested for HIV is unknown) this is in keeping with the average seroprevalence of HIV amongst mentally ill patients (10\%).

In this study mood episodes (mania) with psychotic symptoms were the most common presentation in both the group with a pre-existing primary psychiatric disorder and the group without a primary psychiatric disorder. Comorbid substance abuse was an uncommon finding. Most patients tolerated low doses of typical antipsychotics in combination with a mood stabilizer (valproate being the most common).

Psychiatrists need to be trained in HIV- associated medical disorders and the prescribing of ART, as it is expected that more of these patients will present to our inpatient and outpatient units and an intimate knowledge is necessary in the face of the HIV epidemic.

HIV testing in mentally ill patients should be vigorously debated and the stringent principals for testing in these patients should be reviewed.

The limitations of this study include the small study number as well as not reflecting how many patients were actually tested for HIV during the study period. The results could also be skewed as only a female population was studied.

\section{References}

1. UNAIDS, AIDS epidemic update: December 2009. wwwunaidsorg (last accessed October 2010).

2. The National Department of Health REPORT, THE NATIONAL HIV AND SYPHYLIS PREVALENCE SURVEY SOUTH AFRICA 2007. 2008.

3. Alegria M, Vila D, Train S, Williams S, EI-Bassel N. Epidemiology. Horwath E, Cournos F. Psychotic Disorders. Lagomasino IT, Rodriguez G. HIVIAIDS among women. In: Fernandez F, Ruiz P, Psychiatric aspects of HIVIAIDS. Philadelphia:Lippincott Williams \& Wilkins, 2006:5, 119, 278,282

4. Janse van Rensburg B, Bracken C. Acute psychiatric in-patients tested for HIV status: a clinical profile. South African Psychiatry
Review 2007; 10(2): 83 - 85

5. Joska JA, Kaliski SZ, Benatar SR. Patients with severe mental illness: A new approach to testing for HIV. SAMJ 2008; 98(3): $213-217$.

6. Joska JA, Stein DJ, Flischer AJ. HIVIAIDS and psychiatry: Towards the establishment of a pilot programme for detection and treatment of common mental disorders in people living with HIVIAIDS in Cape Town. SAJP 2008; 14 (4):122 - 124.

7. Olatunji BO, Mimiaga MJ, O'Cleirigh C, Safren SA. A review of treatment studies of depression in HIV. Topics in HIV Medicine 2006 14(3): 112-124.

8. Dube B, Benton T, Cruess DG, Evans DL. Neuropsychiatric manifestations of HIV infection and AIDS. J Psychiatry NeurosCi 2005; 30(4): $237-245$

9. Walton S. HIV and psychiatry. JDA 2000; 3(1):27 - 31 .

10. Buhrich N, Cooper DA, Freed E. HIV Infection Associated with Symptoms Indistinguishable from Functional Psychosis. British Journal of Psychiatry 1998; 152: 649- 653.

11. Davis HF, Skolasky RK, Selnes OA, Burgess DM, MCArthur JC. Assessing HIV-Associated Dementia: Modified HIV Dementia Scale Versus the Grooved Pegboard. AIDS Read 2002; 12(1): 29-38.

12. Robertson KR, Sacktor N, Hall C, Price R, Modi G, Birbeck G. HIV \& AIDS Treatment in Practice \# 81, 2007; Part 11: 1-6

13. Susser E, Colson PHP, Jandorf L, Berkman A, Lavelle J, Fennig S et al. HIV Infection Among Young Adults With Psychotic Disorders. AM J Psychiatry 1997; 154(6): 864 -866.

14. Sewell DD, Jeste DV, Atkinson JH, Heaton RK, Hesselink JR, Wiley C et al. HIV-Associated Psychosis: A Study of 20 Cases. Am J Psychiatry 1994; 151 (2): 237 - 242

15. Halstead S, Riccio M, Harlow P, Oretti R, Thompson C. Psychosis Associated with HIV Infection. British Journal of Psychiatry 1988, 153: $618-623$.

16. Harris MJ, Jeste DV, Gleghorn A, Sewell DD. New-Onset Psychosis in HIV-Infected Patients. J Clin Psychiatry 1991; 52(9): 369 - 375.

17. Mashaphu S, Mkize DL. HIV seropositivity in patients with firstepisode psychosis. SAJP 2007; 13(3): 90 - 94.

18. Sall L, Salamon E, Allgulander C, Owe-Larsson B. Psychiatric symptoms and disorders in HIV infected mine workers in South Africa. A retrospective descriptive study of acute first admissions. Afr J Psychiatry 2009; 12 (3): 206 -212.

19. Vitiello B, Burnam MA, Bing EG, Beckman R, Shapiro M. Use of Psychotropic Medications Among HIV-Infected Patients in the United States. Am J Psychiatry 2003; 160: 547 - 554. 